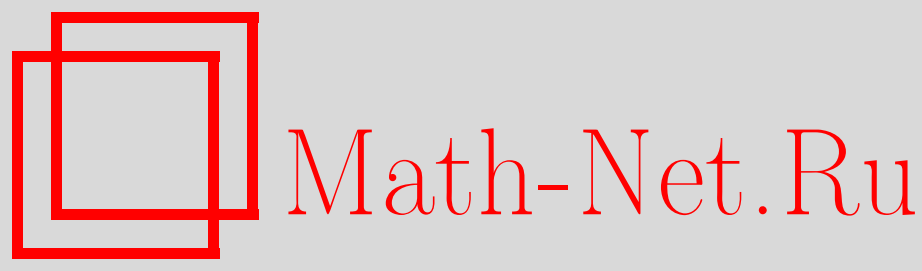

Д. Ю. Письмак, Ю. М. Письмак, Рассеяние электромагнитных волн на плоской поверхности в модели с потенциалом Черна-Саймонса, ТМФ, 2011, том 169, номер 1, 69-78

DOI: https://doi.org/10.4213/tmf6709

Использование Общероссийского математического портала Math-Net.Ru подразумевает, что вы прочитали и согласны с пользовательским соглашением http: //www . mathnet.ru/rus/agreement

Параметры загрузки:

IP : 3.82 .47 .9

26 апреля 2023 г., $16: 26: 13$

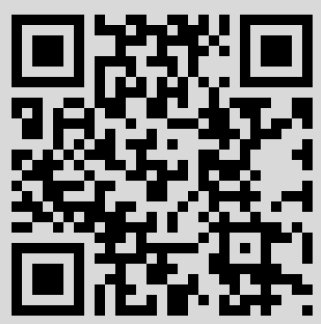




\title{
РАССЕЯНИЕ ЭЛЕКТРОМАГНИТНЫХ ВОЛН НА ПЛОСКОЙ ПОВЕРХНОСТИ В МОДЕЛИ С ПОТЕНЦИАЛОМ ЧЕРНА-САЙМОНСА
}

\begin{abstract}
Потенциал Черна-Саймонса возникает в квантовой электродинамике при построении моделей взаимодействия материальной поверхности с фотонным полем, если наложить требования локальности, калибровочной инвариантности и перенормируемости. В рамках такой модели рассмотрена задача рассеяния электромагнитных волн на плоскости.
\end{abstract}

Ключевые слова: эффект Казимира, электродинамика в пространстве с макроскопическими неоднородностями, потенциал Черна-Саймонса, рассеяние волн.

\section{1. ВВЕДЕНИЕ}

Хорошо известно, что состояние квантовополевой системы зависит от воздействия на нее внешнего фонового поля. С другой стороны, квантовые флуктуации вакуума вносят существенные поправки в классическую картину взаимодействия макроскопических материальных объектов. В 1948 г. Казимир показал, что квантовые флуктуации вызывают притяжение между двумя параллельными пластинами незаряженного конденсатора [1]. Теоретическое предсказание этого явления, названного эффектом Казимира, хорошо согласуется с современными экспериментальными данными [2]-[4]. Характерный масштаб для эффекта Казимира - расстояние $10 \div 1000$ нм. На таких расстояниях существенными оказываются как классические, так и квантовые свойства системы, и это требует разработки специальных методов исследования. Знание законов нанофизики важно не только для более полного понимания природы физических явлений, в настоящее время оно становится также необходимым и для решения различных практических задач в бурно развивающихся направлениях по созданию новых технических устройств микромеханики, разработки нано- и биотехнологий.

На данный момент существует большое число теоретических работ, в которых рассматривается эффект Казимира (см., например, [5]). Однако, интересуясь только

* Санкт-Петербургский государственный университет, Санкт-Петербург, Россия. E-mail: dashap2@gmail.com, ypismak@yahoo.com 
некоторыми его особыми аспектами, многие авторы производят вычисления в упрощенных моделях. Обычно предполагают, что специфика квантовой электродинамики (КЭД) не существенна, и наиболее важные особенности эффекта Казимира могут быть исследованы в рамках свободного квантового скалярного поля с фиксированными граничными условиями или с $\delta$-функцией в качестве потенциала.

Используя такие методы, можно получить количественное описание некоторой характеристики эффекта Казимира, но они неприменимы для исследований в рамках одной и той же модели других явлений, возникающих из-за взаимодействия полей КЭД с классическим фоновым полем (дефектом). Для построения такой модели можно использовать подход Симанзика, в котором к обычному действию квантовой теории поля добавляется действие дефекта, сосредоточенное в области пространства, занятого материальным телом.

Взаимодействие сингулярного внешнего поля, сосредоточенного на двумерной поверхности в трехмерном пространстве, с фотонным полем оказывается полностью определено формой поверхности (дефекта) и ограничениями, вытекающими из основных принципов КЭД (калибровочная инвариантность, локальность, перенормируемость). При этом в действии дефекта содержится только один безразмерный параметр - константа взаимодействия материала поверхности с фотонным полем [6]. Оказывается, что сила Казимира существенно зависит от этого параметра и при определенных его значениях может стать отталкивающей. Кроме того, модель предсказывает необычные эффекты взаимодействия зарядов и токов с материальной плоскостью [6]. В работе [7] с помощью этой модели проведены расчеты потенциала Казимира-Полдера для нейтрального атома, находящегося вблизи плоской поверхности. В настоящей работе данная модель используется для исследования процесса рассеяния электромагнитных волн на плоскости.

\section{2. ПОСТАНОВКА ЗАДАЧИ}

Рассмотрим модель фотонного поля, взаимодействующего с материальной плоскостью. Для общего случая двумерной поверхности, заданной уравнением $\Phi(x)=0$, функционал действия модели имеет вид

$$
S(A)=-\frac{1}{4} F_{\mu \nu} F^{\mu \nu}+S_{\Phi}(A)
$$

где

$$
S_{\Phi}(A)=\frac{a}{2} \int \varepsilon^{\lambda \mu \nu \rho} \partial_{\lambda} \Phi(x) A_{\mu}(x) F_{\nu \rho}(x) \delta(\Phi(x)) d x, \quad F_{\mu \nu}=\partial_{\mu} A_{\nu}-\partial_{\nu} A_{\mu}
$$

Вариационная производная действия дефекта, сосредоточенного на поверхности, записывается как

$$
\frac{\delta S_{\Phi}(A)}{\delta A_{\nu}(y)}=a \varepsilon^{\mu \nu \eta \rho} \partial_{\mu} \Phi(y) F_{\eta \rho}(y) \delta(\Phi(y)) .
$$

Для плоскости $x_{3}=0$ уравнения Эйлера-Лагранжа рассматриваемой модели представляют собой модифицированные уравнения Максвела:

$$
\frac{\delta S(A)}{\delta A_{\nu}}=\partial_{\mu} F^{\mu \nu}+a \varepsilon^{3 \nu \sigma \rho} F_{\sigma \rho} \delta\left(x_{3}\right)=0 .
$$


Мы решим их, воспользовавшись преобразованием Фурье по координатам $x_{0}, x_{1}, x_{2}$ для вектор-потенциала $A_{\mu}$ :

$$
A_{\mu}(x)=\frac{1}{(2 \pi)^{3 / 2}} \int e^{i \bar{p} \bar{x}} A_{\mu}\left(\bar{p}, x_{3}\right) d \bar{p}, \quad A_{\mu}\left(\bar{p}, x_{3}\right)=\frac{1}{(2 \pi)^{3 / 2}} \int e^{-i \bar{p} \bar{x}} A_{\mu}(x) d \bar{x} .
$$

Здесь и в дальнейшем мы используем обозначение $\bar{p}$ для вектора $\bar{p}=\left(p_{0}, p_{1}, p_{2}\right)$, $\bar{p}^{2}=p_{0}^{2}-p_{1}^{2}-p_{2}^{2}, \bar{p} \bar{x}=p_{0} x_{0}-p_{1} x_{1}-p_{2} x_{2}$. Для поля $A\left(\bar{p}, x_{3}\right)$ уравнения (1) записываются в виде

$$
\begin{array}{ll}
\nu=3: & \bar{p}^{2} A^{3}+\partial^{3} i \bar{p} \bar{A}=0 \\
\nu \neq 3: & \left(-\bar{p}^{2}-\partial_{3}^{2}\right) A^{\nu}-i p^{\nu}\left(i \bar{p} \bar{A}-\partial_{3} A_{3}\right)+2 a \varepsilon^{3 \nu \eta \rho} i p_{\eta} A_{\rho} \delta\left(x_{3}\right)=0 .
\end{array}
$$

В силу второго равенства в (2) условие вещественности $A_{\mu}(x)=A_{\mu}^{*}(x)$ вектор-потенциала $A_{\mu}(x)$ для $A\left(\bar{p}, x_{3}\right)$ имеет вид $A^{*}\left(\bar{p}, x_{3}\right)=A\left(-\bar{p}, x_{3}\right)$. Воспользовавшись этим соотношением, можно получить интегральное представление

$$
\begin{aligned}
A_{\mu}(x) & =\frac{1}{(2 \pi)^{3 / 2}} \int \theta\left(p_{0}\right)\left[e^{i \bar{p} \bar{x}} A_{\mu}\left(\bar{p}, x_{3}\right)+e^{-i \bar{p} \bar{x}} A_{\mu}^{*}\left(\bar{p}, x_{3}\right)\right] d \bar{p}= \\
& =\frac{1}{(2 \pi)^{3 / 2}} 2 \operatorname{Re} \int \theta\left(p_{0}\right)\left[e^{i \bar{p} \bar{x}} A_{\mu}\left(\bar{p}, x_{3}\right)\right] d \bar{p},
\end{aligned}
$$

в котором вещественность $A_{\mu}(x)$ очевидна.

\section{3. ВЫБОР КАЛИБРОВКИ}

Уравнение Эйлера-Лагранжа (1) для рассматриваемой задачи инвариантно относительно калибровочного преобразования $A_{\mu}(x) \rightarrow A_{\mu}(x)+\partial_{\mu} \varphi(x)$, поэтому решение уравнения (1) находится с точностью до калибровочного преобразования, и мы можем при его построении зафиксировать калибровку. Мы будем проводить расчеты в темпоральной калибровке

$$
A_{0}=0
$$

Воспользовавшись калибровочным условием (6), перепишем уравнения (3), (4) в виде

$$
\begin{aligned}
\bar{p}^{2} A^{3}-\partial^{3}\left(i p_{1} A_{1}+i p_{2} A_{2}\right) & =0, \\
p^{0}\left(i p_{1} A_{1}+i p_{2} A_{2}+\partial_{3} A_{3}\right)-2 a\left(p_{1} A_{2}-p_{2} A_{1}\right) \delta\left(x_{3}\right) & =0 \\
\left(-\bar{p}^{2}-\partial_{3}^{2}\right) A^{1}+i p^{1}\left(i p_{1} A_{1}+i p_{2} A_{2}+\partial_{3} A_{3}\right)+2 a i p_{0} A_{2} \delta\left(x_{3}\right) & =0 \\
\left(-\bar{p}^{2}-\partial_{3}^{2}\right) A^{2}+i p^{2}\left(i p_{1} A_{1}+i p_{2} A_{2}+\partial_{3} A_{3}\right)-2 a i p_{0} A_{1} \delta\left(x_{3}\right) & =0 .
\end{aligned}
$$

Положим

$$
i p_{1} A_{1}+i p_{2} A_{2}+\partial_{3} A_{3}=\alpha,\left.\quad A_{1}\right|_{x_{3}=0}=a_{1},\left.\quad A_{2}\right|_{x_{3}=0}=a_{2} .
$$

Тогда из уравнения (8) следует, что

$$
\alpha=2 a \frac{p_{1} a_{2}-p_{2} a_{1}}{p_{0}} \delta\left(x_{3}\right),
$$


и в силу уравнений $(9),(10)$ поля $A_{1}, A_{2}$ удовлетворяют уравнениям

$$
\left(\bar{p}^{2}+\partial_{3}^{2}\right) A_{i}+c_{i} \delta\left(x_{3}\right)=0, \quad i=1,2,
$$

в которых

$$
c_{1} \equiv-\frac{2 i a}{p_{0}}\left[\left(p_{1}^{2}-p_{0}^{2}\right) a_{2}-p_{1} p_{2} a_{1}\right], \quad c_{2} \equiv \frac{2 i a}{p_{0}}\left[\left(p_{2}^{2}-p_{0}^{2}\right) a_{1}-p_{1} p_{2} a_{2}\right] .
$$

Таким образом, в рассматриваемом нами случае темпоральной калибровки нам нужно найти решения двух однотипных дифференциальных уравнений (12).

\section{4. РЕШЕНИЕ УРАВНЕНИЯ $\partial_{T}^{2} \psi+P^{2} \psi+C \delta(T)=0$}

Чтобы найти $A\left(\bar{p}, x_{3}\right)$, решим вначале вспомогательную задачу. Найдем общее решение уравнения

$$
\partial_{t}^{2} \psi+p^{2} \psi+c \delta(t)=0
$$

Пусть

$$
f(t) \equiv \frac{c}{2 p i}\left[\theta(t) e^{-i p t}+\theta(-t) e^{i p t}\right]=\frac{c e^{-i p|t|}}{2 p i} .
$$

Продифференцировав эту функцию по $t$, получим

$$
\begin{aligned}
f^{\prime}(t) & =-\frac{c}{2}\left[\theta(t) e^{-i p t}-\theta(-t) e^{i p t}\right], \\
f^{\prime \prime}(t) & =\frac{c p i}{2}\left[\theta(t) e^{-i p t}+\theta(-t) e^{i p t}\right]-c \delta(t) .
\end{aligned}
$$

Таким образом, функция $f(t)$ является одним из решений уравнения (13). Однородное уравнение $\partial_{t}^{2} \varphi+p^{2} \varphi=0$ имеет решение $\varphi(t)=d_{1} e^{i p t}+d_{2} e^{-i p t}$, где $d_{1}, d_{2}-$ произвольные постоянные. Следовательно, общим решением уравнения (13) является

$$
\psi(t)=d_{1} e^{i p t}+d_{2} e^{-i p t}+\frac{c e^{-i p|t|}}{2 p i},
$$

где $d_{1}, d_{2}$ - произвольные постоянные. Полученный результат, как мы покажем далее, позволяет найти решение системы уравнений (7)-(10) в явном виде.

\section{5. РЕШЕНИЕ УРАВНЕНИЙ ЭЙЛЕРА-ЛАГРАНЖА}

Так как функция (15) является общим решением уравнения (13), мы можем записать общее решение уравнений для полей $A_{1}, A_{2}$ в виде

$$
\begin{aligned}
& A_{1}\left(\bar{p}, x_{3}\right)=d_{1}^{(1)} e^{i \rho x_{3}}+d_{2}^{(1)} e^{-i \rho x_{3}}+\frac{c_{1} e^{-i \rho\left|x_{3}\right|}}{2 i \rho} \\
& A_{2}\left(\bar{p}, x_{3}\right)=d_{1}^{(2)} e^{i \rho x_{3}}+d_{2}^{(2)} e^{-i \rho x_{3}}+\frac{c_{2} e^{-i \rho\left|x_{3}\right|}}{2 i \rho}
\end{aligned}
$$

где $\rho \equiv \sqrt{\bar{p}^{2}}$. Нетрудно убедиться, что из предположения ограниченности $A_{j}\left(x_{3}, \bar{p}\right)$ при любых значениях $x_{3}$ следует, что $\bar{p}^{2}>0$, поэтому мы рассмотрим только случай $\rho>0$. Поле $A_{3}$ находится непосредственно из уравнения (7):

$$
A_{3}\left(x_{3}, \bar{p}\right)=d_{1}^{(3)} e^{i \rho x_{3}}+d_{2}^{(3)} e^{-i \rho x_{3}}+\epsilon\left(x_{3}\right) \frac{c_{3} e^{-i \rho\left|x_{3}\right|}}{2 i \rho},
$$


где

$$
d_{1}^{(3)}=\frac{1}{\rho}\left(p_{1} d_{1}^{(1)}+p_{2} d_{1}^{(2)}\right), \quad d_{2}^{(3)}=-\frac{1}{\rho}\left(p_{1} d_{2}^{(1)}+p_{2} d_{2}^{(2)}\right), \quad c_{3}=-\frac{1}{\rho}\left(p_{1} c_{1}+p_{2} c_{2}\right)
$$

и $\epsilon\left(x_{3}\right) \equiv x_{3} /\left|x_{3}\right|$.

Положив $x_{3}=0$ в $(16),(17)$ и воспользовавшись обозначениями (11), мы получим соотношения

$$
a_{j}=d_{1}^{(j)}+d_{2}^{(j)}+\frac{c_{j}}{2 i \rho}, \quad j=1,2 .
$$

Пусть $D_{j}=\left(d_{1}^{(j)}+d_{2}^{(j)}\right) \rho, j=1,2$. Тогда система линейных уравнений для $a_{1}, a_{2}$ запишется в виде

$$
\begin{aligned}
& a_{1}\left(p_{0} \rho-a p_{1} p_{2}\right)+a a_{2}\left(p_{1}^{2}-p_{0}^{2}\right)=D_{1} p_{0}, \\
& a_{2}\left(p_{0} \rho+a p_{1} p_{2}\right)-a a_{1}\left(p_{2}^{2}-p_{0}^{2}\right)=D_{2} p_{0} .
\end{aligned}
$$

Решив ее, получим

$$
\begin{aligned}
& a_{1}=\frac{a D_{2}\left(p_{0}^{2}-p_{1}^{2}\right)+D_{1}\left(a p_{1} p_{2}+p_{0} \rho\right)}{p_{0} \rho^{2}\left(a^{2}+1\right)} \\
& a_{2}=-\frac{a D_{1}\left(p_{0}^{2}-p_{2}^{2}\right)+D_{2}\left(a p_{1} p_{2}-p_{0} \rho\right)}{p_{0} \rho^{2}\left(a^{2}+1\right)}
\end{aligned}
$$

и

$$
\begin{aligned}
& c_{1}=-\frac{2 a i\left[D_{1}\left(a p_{0} \rho-p_{1} p_{2}\right)-D_{2}\left(p_{0}^{2}-p_{1}^{2}\right)\right]}{p_{0} \rho\left(a^{2}+1\right)}, \\
& c_{2}=-\frac{2 a i\left[D_{2}\left(a p_{0} \rho+p_{1} p_{2}\right)+D_{1}\left(p_{0}^{2}-p_{2}^{2}\right)\right]}{p_{0} \rho\left(a^{2}+1\right)} .
\end{aligned}
$$

Положив

$$
\begin{gathered}
\vec{A}=\left(A_{1}, A_{2}, A_{3}\right), \quad \vec{a}=\left(a_{1}, a_{2}, a_{3}\right), \quad \vec{f}=\frac{1}{2 i \rho}\left(c_{1}, c_{2}, c_{3}\right), \\
\overrightarrow{d_{j}}=\left(d_{j}^{(1)}, d_{j}^{(2)}, d_{j}^{(3)}\right), \quad j=1,2,
\end{gathered}
$$

представим формулы (16)-(18) в компактной форме

$$
\vec{A}\left(\bar{p}, x_{3}\right)=\vec{d}_{1}(\bar{p}) e^{i \rho x_{3}}+\vec{d}_{2}(\bar{p}) e^{-i \rho x_{3}}+R\left(x_{3}\right) \vec{f}(\bar{p}) e^{-i \rho\left|x_{3}\right|},
$$

где $R\left(x_{3}\right)$ - диагональная матрица с элементами $R_{11}\left(x_{3}\right)=R_{22}\left(x_{3}\right)=1, R_{33}\left(x_{3}\right)=$ $\epsilon\left(x_{3}\right)$. Таким образом, воспользовавшись соотношениями (5), (21), мы получаем следующее представление решения уравнений Эйлера-Лагранжа для рассматриваемой модели:

$$
\begin{aligned}
& \vec{A}(x)= \frac{\theta\left(x_{3}\right)}{(2 \pi)^{3 / 2}} 2 \operatorname{Re} \int \theta\left(p_{0}\right)\left\{\vec{d}_{1}(\bar{p}) e^{i\left(\bar{p} \bar{x}+\rho x_{3}\right)}+\left[\vec{d}_{2}(\bar{p})+\vec{f}(\bar{p})\right] e^{i\left(\bar{p} \bar{x}-\rho x_{3}\right)}\right\} d \bar{p}+ \\
&+\frac{\theta\left(-x_{3}\right)}{(2 \pi)^{3 / 2}} 2 \operatorname{Re} \int \theta\left(p_{0}\right)\left\{\left[\vec{d}_{1}(\bar{p})+T \vec{f}(\bar{p})\right] e^{i\left(\bar{p} \bar{x}+\rho x_{3}\right)}+\vec{d}_{2}(\bar{p}) e^{i\left(\bar{p} \bar{x}-\rho x_{3}\right)}\right\} d \bar{p} .
\end{aligned}
$$

Здесь $T=R(-1)$ диагональная матрица с элементами $T_{11}\left(x_{3}\right)=T_{22}\left(x_{3}\right)=1$, $T_{33}\left(x_{3}\right)=-1$. Первые слагаемые в подынтегральных выражениях в (22) описывают волны, движущиеся в отрицательном направлении третьей оси, а вторые в положительном. 


\section{6. РАССЕЯНИЕ ВОЛН НА ПЛОСКОСТИ}

Для задачи рассеяния волны с волновым вектором $\vec{k}=\left(k_{1}, k_{2}, k_{3}\right)$, падающей на плоскость из полупространства с отрицательной координатой $x_{3}$, мы должны иметь в полупространстве $x_{3}>0$ только проходящую волну, движущуюся от плоскости $x_{3}=0$ в положительном направлении третьей оси. Следовательно, в (22) мы должны положить $\vec{d}_{1}=0$. В результате получим

$$
\begin{aligned}
\vec{A}(x)= & \frac{\theta\left(x_{3}\right)}{(2 \pi)^{3 / 2}} 2 \operatorname{Re} \int \theta\left(p_{0}\right) \vec{A}_{\mathrm{tr}}(\bar{p}) e^{i\left(\bar{p} \bar{x}-\rho x_{3}\right)} d \bar{p}+ \\
& +\frac{\theta\left(-x_{3}\right)}{(2 \pi)^{3 / 2}} 2 \operatorname{Re} \int \theta\left(p_{0}\right)\left\{\vec{A}_{\mathrm{r}} e^{i\left(\bar{p} \bar{x}+\rho x_{3}\right)}+\vec{A}_{\mathrm{in}} e^{i\left(\bar{p} \bar{x}-\rho x_{3}\right)}\right\} d \bar{p},
\end{aligned}
$$

где векторные амплитуды $\vec{A}_{\text {in }}(\bar{p}), \vec{A}_{\mathrm{r}}(\bar{p}), \vec{A}_{\mathrm{tr}}(\bar{p})$ падающей, отраженной и проходящей волн записываются в виде

$$
\vec{A}_{\text {in }}(\bar{p})=\vec{d}_{2}(\bar{p}), \quad \vec{A}_{\mathrm{r}}(\bar{p})=T \vec{f}(\bar{p}), \quad \vec{A}_{\text {tr }}(\bar{p})=\vec{d}_{2}(\bar{p})+\vec{f}(\bar{p})
$$

и тем самым удовлетворяют соотношению

$$
\overrightarrow{A_{\mathrm{r}}}=T\left(\vec{A}_{\mathrm{tr}}-\vec{A}_{\mathrm{in}}\right) .
$$

Таким образом, векторная амплитуда отраженной волны получается из разности амплитуд падающей и проходящей волн изменением в ней знака у третьей компоненты. Из равенства (24) следует, что имеются только две независимые векторные амплитуды волн, которые определяют третью амплитуду.

\section{7. СОБСТВЕННЫЕ МОДЫ}

Собственными модами мы назовем волны, для которых амплитуды падающей и проходящей волн пропорциональны друг другу:

$$
\vec{A}_{\text {tr }}=\lambda \vec{A}_{\text {in }} .
$$

Для них из формул (24), (25) следует, что

$$
\vec{A}_{\mathrm{r}}=(\lambda-1) T \vec{A}_{\text {in }}, \quad a_{1}=\lambda d_{2}^{(1)}, \quad a_{2}=\lambda d_{2}^{(2)} .
$$

Воспользовавшись (19), (20), найдем соотношения, которым в силу двух последних равенств в (26) должны удовлетворять $d_{2}^{(1)}, d_{2}^{(2)}$ :

$$
\begin{aligned}
& \lambda d_{2}^{(1)}=\frac{a d_{2}^{(2)}\left(p_{0}^{2}-p_{1}^{2}\right)+d_{2}^{(1)}\left(a p_{1} p_{2}+p_{0} \rho\right)}{p_{0} \rho\left(a^{2}+1\right)} \\
& \lambda d_{2}^{(2)}=-\frac{a d_{2}^{(1)}\left(p_{0}^{2}-p_{2}^{2}\right)+d_{2}^{(2)}\left(a p_{1} p_{2}-p_{0} \rho\right)}{p_{0} \rho\left(a^{2}+1\right)} .
\end{aligned}
$$

Данная система уравнений имеет ненулевые решения $d_{2}^{(1)}, d_{2}^{(2)}$, если

$$
\left(a^{2}+1\right) \lambda^{2}-2 \lambda+1=0 .
$$


Это уравнение имеет два решения

$$
\lambda_{1} \equiv \frac{i}{i-a}, \quad \lambda_{2} \equiv \frac{i}{i+a} .
$$

Таким образом, имеются две собственные моды, для которых

$$
\begin{array}{ll}
\lambda=\lambda_{1}, & \overrightarrow{\mathcal{A}}_{\text {in }}^{(1)}=g_{1} \vec{V}_{1}, \\
\lambda=\lambda_{2}, & \overrightarrow{\mathcal{A}}_{\text {in }}^{(2)}=g_{2} \vec{V}_{2},
\end{array}
$$

где

$$
\begin{aligned}
& \vec{V}_{1} \equiv\left(p_{0}^{2}-p_{1}^{2},-i p_{0} \rho-p_{1} p_{2}, i p_{0} p_{2}-p_{1} \rho\right), \\
& \vec{V}_{2} \equiv\left(p_{0}^{2}-p_{1}^{2}, i p_{0} \rho-p_{1} p_{2},-i p_{0} p_{2}-p_{1} \rho\right)
\end{aligned}
$$

и $g_{1}, g_{2}-$ произвольные функции от $p_{0}, p_{1}, p_{2}$. Используя эти обозначения, мы можем записать векторные амплитуды $\overrightarrow{\mathcal{A}_{\text {in }}}(\bar{p}), \overrightarrow{\mathcal{A}_{\mathrm{r}}}(\bar{p}), \overrightarrow{\mathcal{A}_{\mathrm{tr}}}(\bar{p})$ собственных мод падающей, отраженной и проходящей волн в следующем виде:

$\overrightarrow{\mathcal{A}}_{\mathrm{in}}^{(j)}=g_{j}(\bar{p}) \vec{V}_{j}(\bar{p}), \quad \overrightarrow{\mathcal{A}}_{\mathrm{r}}^{(j)}=g_{j}(\bar{p}) K_{\mathrm{r}}^{(j)} T \vec{V}_{j}(\bar{p}), \quad \overrightarrow{\mathcal{A}}_{\mathrm{tr}}^{(j)}=g_{j}(\bar{p}) K_{\mathrm{tr}}^{(j)} \vec{V}_{j}(\bar{p}), \quad j=1,2$.

Здесь мы использовали обозначения

$$
K_{\mathrm{r}}^{(1)}=\frac{i a+a^{2}}{1+a^{2}}, \quad K_{\mathrm{r}}^{(2)}=\frac{-i a+a^{2}}{1+a^{2}}, \quad K_{\mathrm{tr}}^{(1)}=\frac{1-i a}{1+a^{2}}, \quad K_{\mathrm{tr}}^{(2)}=\frac{1+i a}{1+a^{2}} .
$$

Полученные нами характеристики собственных мод удовлетворяют соотношениям $\vec{V}_{2}=\vec{V}_{1}^{*}, K_{\mathrm{tr}}^{(2)}=K_{\mathrm{tr}}^{(1) *}, K_{\mathrm{r}}^{(2)}=K_{\mathrm{r}}^{(1) *}$.

\section{8. РАССЕЯНИЕ ПЛОСКИХ ВОЛН}

Выберем в качестве собственных мод плоские волны с векторными амплитудами $\overrightarrow{\mathcal{A}}_{\text {in }}^{(j)}(\bar{p})=\vec{V}_{j}(\bar{p}), j=1,2$. Мы рассмотрим также их линейную комбинацию $\overrightarrow{\mathcal{A}_{\text {in }}}=$ $C_{1} \overrightarrow{\mathcal{A}}_{\mathrm{in}}^{(1)}+C_{2} \overrightarrow{\mathcal{A}}_{\mathrm{in}}^{(2)}$ с коэффициентами $C_{1}, C_{2}$. Для векторных амплитуд $\mathcal{A}_{\mathrm{r}}(\bar{p}), \mathcal{A}_{\mathrm{tr}}(\bar{p})$ отраженной и проходящей волн мы имеем

$$
\overrightarrow{\mathcal{A}_{\mathrm{r}}}=C_{1} K_{\mathrm{r}}^{(1)} T \vec{V}_{1}+C_{2} T K_{\mathrm{r}}^{(1) *} \vec{V}_{1}^{*}, \quad \overrightarrow{\mathcal{A}}_{\mathrm{tr}}=C_{1} K_{\mathrm{tr}}^{(1)} \vec{V}_{1}+C_{2} K_{\mathrm{tr}}^{(1) *} \vec{V}_{1}^{*} .
$$

Вектор $\vec{V}_{1}$ можно представить в виде

$$
\vec{V}_{1}=\vec{U}_{1}+i \vec{U}_{2}, \quad \vec{U}_{1} \equiv\left(p_{0}^{2}-p_{1}^{2},-p_{1} p_{2},-p_{1} \rho\right), \quad \vec{U}_{2} \equiv\left(0,-p_{0} \rho, p_{0} p_{2}\right),
$$

поэтому

$$
\begin{array}{lll}
K_{r}^{(1)} \vec{V}_{1}=\frac{a}{1+a^{2}}\left(\vec{Y}_{1}+i \vec{Y}_{2}\right), & \vec{Y}_{1} \equiv a \vec{U}_{1}-\vec{U}_{2}, & \vec{Y}_{2} \equiv a \vec{U}_{2}+\vec{U}_{1}, \\
K_{\mathrm{tr}}^{(1)} \vec{V}_{1}=\frac{1}{1+a^{2}}\left(\vec{Z}_{1}+i \vec{Z}_{2}\right), & \vec{Z}_{1} \equiv \vec{U}_{1}+a \vec{U}_{2}=\vec{Y}_{2}, & \vec{Z}_{2} \equiv \vec{U}_{2}-a \vec{U}_{1}=-\vec{Y}_{1} .
\end{array}
$$

Следовательно,

$$
\begin{aligned}
\overrightarrow{\mathcal{A}}_{\mathrm{in}} & =\left(C_{1}+C_{2}\right) \vec{U}_{1}+i\left(C_{1}-C_{2}\right) \vec{U}_{2}, \\
\overrightarrow{\mathcal{A}}_{\mathrm{r}} & =\frac{a}{1+a^{2}}\left[\left(C_{1}+C_{2}\right) T \vec{Y}_{1}+i\left(C_{1}-C_{2}\right) T \vec{Y}_{2}\right], \\
\overrightarrow{\mathcal{A}}_{\mathrm{tr}} & =\frac{1}{1+a^{2}}\left[\left(C_{1}+C_{2}\right) \vec{Z}_{1}+i\left(C_{1}-C_{2}\right) \vec{Z}_{2}\right] .
\end{aligned}
$$


Если положить

$\kappa_{1}=\left|C_{1}+C_{2}\right|, \quad \kappa_{2}=\left|C_{1}-C_{2}\right|, \quad \phi_{1}=-i \ln \frac{C_{1}+C_{2}}{\left|C_{1}+C_{2}\right|}, \quad \phi_{2}=-i \ln \frac{C_{2}-C_{1}}{\left|C_{2}-C_{1}\right|}$,

то вектор-потенциалы рассматриваемых нами плоских волн запишутся в виде

$$
\begin{aligned}
& \vec{A}_{\text {in }}(\bar{p}, x)=\sigma \operatorname{Re} \overrightarrow{\mathcal{A}_{\text {in }}}(\bar{p}) e^{i\left(\bar{p} \bar{x}-\rho x_{3}\right)}=\kappa_{1} \alpha_{\text {in }} \vec{U}_{1}-\kappa_{2} \beta_{\text {in }} \vec{U}_{2}, \\
& \overrightarrow{A_{\mathrm{r}}}(\bar{p}, x)=\sigma \operatorname{Re} \overrightarrow{\mathcal{A}}_{\mathrm{r}}(\bar{p}) e^{i\left(\bar{p} \bar{x}+\rho x_{3}\right)}=\kappa_{1} \alpha_{\mathrm{r}} T \vec{Y}_{1}-\kappa_{2} \beta_{\mathrm{r}} T \vec{Y}_{2}, \\
& \vec{A}_{\mathrm{tr}}(\bar{p}, x)=\sigma \operatorname{Re} \overrightarrow{\mathcal{A}}_{\mathrm{tr}}(\bar{p}) e^{i\left(\bar{p} \bar{x}-\rho x_{3}\right)}=\kappa_{1} \alpha_{\mathrm{tr}} \vec{Z}_{1}-\kappa_{2} \beta_{\mathrm{tr}} \vec{Z}_{2} \text {, }
\end{aligned}
$$

где $\sigma \equiv 2(2 \pi)^{-3 / 2}$,

$$
\begin{aligned}
\alpha_{\mathrm{in}} & =\sigma \cos \left(\bar{p} \bar{x}-\rho x_{3}+\phi_{1}\right), & \beta_{\mathrm{in}} & =\sigma \sin \left(\bar{p} \bar{x}-\rho x_{3}+\phi_{2}\right), \\
\alpha_{\mathrm{r}} & =\frac{a \sigma}{1+a^{2}} \cos \left(\bar{p} \bar{x}+\rho x_{3}+\phi_{1}\right), & \beta_{\mathrm{r}} & =\frac{a \sigma}{1+a^{2}} \sin \left(\bar{p} \bar{x}+\rho x_{3}+\phi_{2}\right), \\
\alpha_{\mathrm{tr}} & =\frac{a}{1+a^{2}} \alpha_{\mathrm{in}}, & \beta_{\mathrm{tr}} & =\frac{a}{1+a^{2}} \beta_{\mathrm{in}} .
\end{aligned}
$$

В рассматриваемой нами калибровке напряженность электрического поля $\vec{E}$ совпадает с производной по $x_{0}$ от вектор-потенциала $\vec{A}$ :

$$
\begin{aligned}
\vec{E}_{\mathrm{in}} & =\sigma \operatorname{Re}\left(i p_{0} \overrightarrow{\mathcal{A}}_{\mathrm{in}}(\bar{p}) e^{i\left(\bar{p} \bar{x}-\rho x_{3}\right)}\right)=-p_{0}\left(\kappa_{1} \beta_{\mathrm{in}} \vec{U}_{1}+\kappa_{2} \alpha_{\mathrm{in}} \vec{U}_{2}\right), \\
\vec{E}_{\mathrm{r}} & =\sigma \operatorname{Re}\left(i p_{0} \overrightarrow{\mathcal{A}}_{\mathrm{r}}(\bar{p}) e^{i\left(\bar{p} \bar{x}+\rho x_{3}\right)}\right)=-p_{0}\left(\kappa_{1} \beta_{\mathrm{r}} T \vec{Y}_{1}+\kappa_{2} \alpha_{\mathrm{r}} T \vec{Y}_{2}\right), \\
\vec{E}_{\mathrm{tr}} & =\sigma \operatorname{Re}\left(i p_{0} \overrightarrow{\mathcal{A}}_{\mathrm{tr}}(\bar{p}) e^{i\left(\bar{p} \bar{x}-\rho x_{3}\right)}\right)=-p_{0}\left(\kappa_{1} \beta_{\mathrm{tr}} \vec{Z}_{1}+\kappa_{2} \alpha_{\mathrm{tr}} \vec{Z}_{2}\right) .
\end{aligned}
$$

Магнитное поле вычисляется по формуле $\vec{H}=\vec{\partial} \times \vec{A}$, из которой непосредственно получается следующий результат:

$$
\vec{H}_{\mathrm{in}}=-\frac{\vec{P}_{\mathrm{in}} \times \vec{E}_{\mathrm{in}}}{p_{0}}, \quad \vec{H}_{\mathrm{r}}=-\frac{\vec{P}_{\mathrm{r}} \times \vec{E}_{\mathrm{r}}}{p_{0}}, \quad \vec{H}_{\mathrm{tr}}=-\frac{\vec{P}_{\mathrm{tr}} \times \vec{E}_{\mathrm{tr}}}{p_{0}},
$$

где $\vec{H}_{\mathrm{in}}, \vec{H}_{\mathrm{r}}, \vec{H}_{\mathrm{tr}}$ - векторы напряженностей магнитного поля падающей, отраженной, проходящей волн и $\vec{P}_{\mathrm{in}}=\vec{P}_{\mathrm{tr}}=\left(p_{1}, p_{2}, \rho\right), \vec{P}_{\mathrm{r}}=\left(p_{1}, p_{2},-\rho\right)$. Подставив в (34) векторы напряженностей электрических полей $(33)$, получим

$$
\begin{aligned}
\vec{H}_{\mathrm{in}} & =p_{0}\left(\kappa_{2} \alpha_{\mathrm{in}} \vec{U}_{1}-\kappa_{1} \beta_{\mathrm{in}} \vec{U}_{2}\right), \\
\vec{H}_{\mathrm{r}} & =-p_{0}\left(\kappa_{2} \alpha_{\mathrm{r}} T \vec{Y}_{1}-\kappa_{1} \beta_{\mathrm{r}} T \vec{Y}_{2}\right), \\
\vec{H}_{\mathrm{tr}} & =p_{0}\left(\kappa_{2} \alpha_{\mathrm{tr}} \vec{Z}_{1}-\kappa_{1} \beta_{\mathrm{tr}} \vec{Z}_{2}\right) .
\end{aligned}
$$

Для интенсивностей $I_{\mathrm{in}}, I_{\mathrm{r}}, I_{\mathrm{tr}}$ падающей, отраженной и проходящей волн мы имеем следующие выражения:

$$
I_{\mathrm{in}} \equiv\left|\sigma \overrightarrow{\mathcal{A}}_{\mathrm{in}}(\bar{p}) e^{i\left(\bar{p} \bar{x}-\rho x_{3}\right)}\right|^{2}=\left|\sigma \overrightarrow{\mathcal{A}}_{\mathrm{in}}(\bar{p})\right|^{2}, \quad \vec{I}_{\mathrm{r}}=\left|\sigma \overrightarrow{\mathcal{A}}_{\mathrm{r}}(\bar{p})\right|^{2}, \quad{\overrightarrow{I_{\mathrm{tr}}}}^{2}=\left|\sigma \overrightarrow{\mathcal{A}}_{\mathrm{tr}}(\bar{p})\right|^{2} .
$$

Непосредственно из определений векторов $\vec{U}_{j}, \vec{Y}_{j}, \vec{Z}_{j}(31)$ и матрицы $T$ получаем

$$
\begin{gathered}
\vec{U}_{1} \vec{U}_{2}=\vec{Y}_{1} \vec{Y}_{2}=\vec{Z}_{1} \vec{Z}_{2}=0, \quad \vec{U}_{1} \vec{U}_{1}=\vec{U}_{2} \vec{U}_{2}=p_{0}^{2}\left(p_{0}^{2}-p_{1}^{2}\right), \\
\vec{Y}_{1} \vec{Y}_{1}=\vec{Y}_{2} \vec{Y}_{2}=\vec{Z}_{1} \vec{Z}_{1}=\vec{Z}_{2} \vec{Z}_{2}=p_{0}^{2}\left(p_{0}^{2}-p_{1}^{2}\right)\left(1+a^{2}\right), \quad T T^{\mathrm{T}}=T^{2}=1 .
\end{gathered}
$$


В силу (32), (35), (36) имеем

$$
I_{\mathrm{r}}=\frac{a^{2}}{1+a^{2}} I_{\mathrm{in}}, \quad I_{\mathrm{tr}}=\frac{1}{1+a^{2}} I_{\mathrm{in}}
$$

Следовательно, коэффициенты отражения $K_{\mathrm{r}} \equiv I_{\mathrm{r}} / I_{\mathrm{in}}$ и прохождения $K_{\mathrm{tr}} \equiv I_{\mathrm{tr}} / I_{\mathrm{in}}$ плоской волны при ее рассеянии на плоскости не зависят от частоты, угла падения и выражаются через константу $a$ взаимодействия электромагнитного поля с поверхностью, характеризующую свойства ее материала:

$$
K_{\mathrm{r}}=\frac{a^{2}}{1+a^{2}}, \quad K_{\mathrm{tr}}=\frac{1}{1+a^{2}} .
$$

Рассмотрим движения волн вдоль оси $x_{3}$. В этом случае $p_{1}=p_{2}=0, \rho=p_{0}$ и

$$
\begin{aligned}
\vec{E}_{\mathrm{in}} & =p_{0}^{3}\left(-\kappa_{1} \beta_{\mathrm{in}}, \kappa_{2} \alpha_{\mathrm{in}}, 0\right), \\
\vec{E}_{\mathrm{tr}} & =\frac{p_{0}^{3}}{1+a^{2}}\left(-\kappa_{1} \beta_{\mathrm{in}}+\kappa_{2} \alpha_{\mathrm{in}} a, \kappa_{2} \alpha_{\mathrm{in}}+\kappa_{1} \beta_{\mathrm{in}} a, 0\right), \\
\vec{E}_{\mathrm{r}} & =\frac{a p_{0}^{3}}{1+a^{2}}\left(-\kappa_{1} \beta_{\mathrm{r}} a-\kappa_{2} \alpha_{\mathrm{r}}, \kappa_{2} \alpha_{\mathrm{r}} a-\kappa_{1} \beta_{\mathrm{r}}, 0\right) .
\end{aligned}
$$

Таким образом,

$$
\vec{E}_{\mathrm{tr}}=\frac{1}{1+a^{2}} \vec{E}_{\mathrm{in}}+\frac{a}{1+a^{2}} \vec{Q}, \quad \vec{Q} \equiv p_{0}^{3}\left(\kappa_{2} \alpha_{\mathrm{in}}, \kappa_{1} \beta_{\mathrm{in}}, 0\right),
$$

а при замене в $\vec{E}_{\mathrm{r}}$ знака величины $x_{3}$ на противоположный

$$
\vec{E}_{\mathrm{r}}=\frac{a^{2}}{1+a^{2}} \vec{E}_{\mathrm{in}}-\frac{a}{1+a^{2}} \vec{Q}
$$

Мы видим, что при рассеянии волны, которая движется перпендикулярно плоскости, кроме обычных для процесса рассеяния волн, возникают волны с повернутым на угол $\pi / 2$ вектором напряженности электрического поля $\left(\vec{E}_{\mathrm{in}} \vec{Q}=0\right)$.

\section{9. ЗАКЛЮЧЕНИЕ}

Мы рассмотрели задачу рассеяния электромагнитной волны на плоскости. В рамках модели со взаимодействием Черна-Саймонса двумерной поверхности с фотонным полем в калибровке $A_{0}=0$ получено решение модифицированных уравнений Максвелла, содержащее безразмерный параметр, который описывает свойства материала рассеивающей электромагнитные волны плоскости. Мы построили в явном виде две линейно независимые собственные моды задачи рассеяния, для которых вектор-потенциалы отраженной и проходящей волн в комплексной форме получаются из вектор-потенциала падающей волны умножением на комплексные числа. Получены вектор-потенциалы, электрическое и магнитное поля отраженной и проходящей волн для случая рассеяния плоской волны произвольной поляризации. Коэффициенты прохождения и отражения оказались не зависящими от энергии падающей волны и угла падения. Нами был рассмотрен случай распространения волн в направлении, ортогональном плоскости. Обнаружен поворот вектора поляризации на угол, близкий к $\pi / 2$, у отраженной волны, исчезающей при малой константе 
взаимодействия плоскости с электромагнитным полем. Вектор поляризации исчезающей в пределе бесконечной константы взаимодействия проходящей волны также оказался повернутым по отношению к вектору падающей волны на угол $\pi / 2$. Эти эффекты аналогичны спонтанному нарушению симметрии, при котором нарушающее симметрии внешнее воздействие приводит к изменениям состояния системы, не исчезающим в пределе уменьшения данного воздействия до сколь угодно малой величины. Полученные результаты позволяют надеяться на возможность экспериментальной проверки модели взаимодействия полей КЭД с макроскопическими объектами и определения на основе экспериментальных данных параметров, характеризующих свойства материала.

\section{Список литературы}

[1] H. B. G. Casimir, Proc. Akad. Wet. Amsterdam, 51 (1948), 793-795.

[2] U. Mohideen, A. Roy, Phys. Rev. Lett., 81:21 (1998), 4549-4552, arXiv: physics/9805038; A. Roy, C.-Y. Lin, U. Mohideen, Phys. Rev. D, 60:11 (1999), 111101(R), 5 pp., arXiv: quant-ph/9906062.

[3] B. W. Harris, F. Chen, U. Mohideen, Phys. Rev. A, 62:5 (2000), 052109, 5 pp.

[4] G. Bressi, G. Carugno, R. Onofrio, G. Ruoso, Phys. Rev. Lett., 88:4 (2002), 041804, 4 pp., arXiv: quant-ph/0203002.

[5] K. A. Milton, J. Phys. A, 37:38 (2004), R209-R277, arXiv: hep-th/0406024.

[6] V. N. Markov, Yu. M. Pis'mak, J. Phys. A, 39:21 (2006), 6525-6532, arXiv: hep-th/0606058.

[7] V. N. Marachevsky, Yu. M. Pis'mak, Phys. Rev. D, 81 (2010), 065005, 6 pp., arXiv: 0907.1985. 